\title{
Adipose-Derived Mesenchymal Stem Cells: Past, Present, and Future
}

\author{
Gino Rigotti · Alessandra Marchi · Andrea Sbarbati
}

Published online: 21 April 2009

(C) Springer Science+Business Media, LLC and International Society of Aesthetic Plastic Surgery 2009

It is likely that in the future, the past decade in the history of plastic surgery will be remembered for the introduction of reconstructive therapies based on the use of stem cells. Of the variety of different approaches that have been proposed, those that have been most widely used in recent years, and which therefore seem most promising for the future, are those using adipose-derived mesenchymal stem cells [1]. The current use and the immense promise of stem cells from adipose tissue are due largely to three aspects that make the procedure simple:

1. The possibility of minimally invasive autologous transplants. Adipose-derived mesenchymal stem cells are obtained by lipoaspiration followed by centrifugation. They can be directly injected into areas to be treated immediately after extraction, there in the operating theatre.

2. Cell expansion is not necessary. As said, the cells can be injected immediately after extraction, along with the lipoaspirate that surrounds them. They do not need to be pre-expanded in vitro. To complete the concept, the addition of the lipoaspirate means that what are injected are "stem cell niches" in which the cells are surrounded by a glycoproteic scaffold, including tissue factors that would be eliminated with digestion of the

G. Rigotti $(\bowtie)$

Plastic Surgery Department and Burn Unit,

Ospedale Civile Maggiore, Verona, Italy

e-mail: gino.rigotti@azosp.vr.it

A. Marchi

Regional Center for Breast Reconstruction, Plastic Surgery

Department, Ospedale Civile Maggiore, Verona, Italy

\author{
A. Sbarbati \\ Institute of Anatomy, University of Verona, Verona, Italy
}

matrix if treated with collagenase-an indispensable step if expansion in vitro were necessary.

3. Age is no barrier. The procedure can be carried out at any time in a patient's life since adipose stem cells are abundant even in the elderly and show the ability to repair lesionated tissues highly efficiently.

The efficacy of the technique has been confirmed repeatedly and under a variety of different conditions. How did this use of adipose-derived mesenchymal stem cells originate and develop? And above all, what are the future therapeutic possibilities of this technique?

\section{The Past: Fat as a Filler}

At times, medical research originates from old ideas that were not sufficiently investigated or interpreted in the past. What we are discussing is a good example: The idea of autologous transplants of fatty tissue is not new in plastic surgery. There are publications about the use of fat as a filler that date back to the end of 19th century. The technique of "lipofilling" developed out of these experiences and has been used to treat thousands of patients with a wide range of indications. However, despite its undoubted advantages, the simple injection of fat was not without problems, the most significant being the poor reproducibility of results and the risk of generating "nodules," which can lead to oil cysts or, less frequently, to granulomas. Credit is due to Coleman for directing attention to these limitations, which were linked to the techniques used to extract and inject fat, and for laying the foundations for a more effective use of adipose tissue [2]. His main insight was to understand the importance of a uniform distribution of the lipoaspirate injected into the tissue to be treated, 
avoiding, at all costs, administration in bolus. Then, centrifugation, another technical development introduced by Coleman, significantly reduced the quantity of material to be used, making it possible to increase the predictability and the stability of results. The purification is performed only by mechanical procedures and is mainly based on a centrifugation protocol, which allows for removal of the cell debris and triglycerides that generate lipolitic products with possible toxic activity [3].

Recent studies demonstrate that adipose-derived stem cells show excellent vitality after purification procedures. So after the introduction of these technical modifications, by the end of the last century it was generally accepted that adipose tissue could be used as a filler and that the method was worthy of scientific attention, although with some reservations, because in many surgeons' experiences results were not consistent.

\section{The Present: Fat as a Regenerating Agent}

In recent years, building on the technical premises outlined above, research into adipose-derived mesenchymal stem cells has advanced, and the biological rationale underlying the methodology has been progressively unveiled. In particular, immunophenotypical and molecular characterization of these cells makes it possible nowadays to identify them and compare them with mesenchymal stem cells from other, better-known sources, like the stem cells derived from bone marrow [4]. In the field of surgery, methods have improved, and the union of clinical work-conducted in particular on the outcomes of radiotherapy after breast cancer therapywith basic biological research has gradually effected a radical change in the way adipose tissue is utilized.

It is now known that the action of injected fat is largely independent of the effects of mature adipocytes and is to be attributed mostly to the contribution of mesenchymal stem cells, which are very abundant in the vascular-stromal fraction of adipose tissue so that this fraction has been identified as truly responsible for therapeutic effects. In published studies, this model applied to therapy seemed capable of improving a series of histologic parameters; in particular, it improved the vascularization of tissue, reducing fibrosis, and even improved the trophism of the cutis overlying the lesions, removing any pain [5].

But what are the mechanisms responsible for the efficacy of therapy using a simple "lipoaspirate," albeit with the technical refinements we have described? The answers can be deduced in part from experiments conducted with adipose stem cells and in part from experiments conducted with other types of stem cells. Published studies have demonstrated that these cells have angiogenic, antifibrotic, and anti-inflammatory effects [6]. These effects seem to be at least partly mediated by the release of growth factors. The anti-inflammatory effect on immune cells seems also to be a clearly biological process in that a reduction of the activity of $\mathrm{T}$ lymphocytes takes place. The factors underlying other effects, for example, the analgesic effect, are unclear.

In this context, one phenomenon, which was initially underestimated but whose importance has recently emerged, is the action of the "stem cell niche," which seems stronger than that of the isolated stem cell [7]. The pathologic models used to test modern therapeutic approaches based on adipose-derived mesenchymal stem cells are numerous, and the results all point in the same direction: These approaches have regularly been shown to be effective in the treatment of the outcomes of radiotherapy, Romberg's disease, and sclerodermia; in the correction of scars, especially retracted and/or hypertrophic scars; in breast reconstruction and cosmetic surgery; and in the treatment of cutaneous aging. A meta-analysis of the modern scientific literature would show how adipose stem cells, or rather "stem cell niches," can be used as regenerative agents and nowadays constitute a valid therapeutic option.

\section{The Future: The Rejuvenation (or Rather the Restemming) of Connective Tissues}

Although the use of adipose stem cell niches in plastic surgery is constantly expanding, they probably have not yet revealed their full potential and will continue to surprise us. Clinically, of course, much remains to be done to verify their efficacy in treating pathologies for which they have not yet been tested. At the same time, preclinical research has still to analyze and further explore the biological foundations of this approach. Nevertheless, the future seems bright. New evidence suggests that therapy using adipose stem cell niches may potentially constitute a real treatment for aging-surely the principal problem of contemporary medicine, now that life expectancy has gradually increased from 45 years at the beginning of the 20th century to about 80 years in advanced industrialized countries. This lengthening of the average life expectancy forces an increasing proportion of the population to live with organs that are not diseased, merely old. The histologic basis of this aging process would seem to be the loss of stemness, i.e., a progressive reduction in the number and quality of stem cells. It has now emerged that the anatomical foundation of regenerative mechanisms is the stem cell niche, which is made up of the stem cell itself and a surrounding system of structures capable of regulating its progression toward differentiation, maturity, and the development of tissues. Treatment with adipose stem cell niches conducted directly in the operating 
room, without any digestion of cells or expansion in vitro, seems to be a method particularly suitable for the reconstruction of stem cell niches for other tissues. The therapeutic target is the reconstruction of autologous niches, by simply moving stem cell niches from one connective tissue in which they are abundant into another in which they are scarce. From fatty tissue, where they remain youthful and plentiful, these niches can be transplanted into other tissues and organs where niches are lacking. The process in many ways resembles autologous transplantation of hair follicles in cases of alopecia.

In this context, what most clearly characterizes modern plastic and cosmetic surgery is the search for ever less invasive surgical procedures, along with a tendency toward greater "naturalness" of the operation.

\section{Conclusions}

Our conclusion can be only that techniques based on the use of stem cell niches from adipose tissue are already important in plastic and cosmetic surgery, and promise to be even more so in future. In the coming years the real challenge for surgeons in these fields will be not only to treat lesions but also to develop technologies capable of opposing the processes of normal aging, by acting on the capacity of tissues to restore equilibrium between proliferative and degenerative mechanisms.

\section{References}

1. Schäffler A, Büchler C (2007) Concise review: adipose tissuederived stromal cells-basic and clinical implications for novel cell-based therapies. Stem Cells 25(4):818-827

2. Coleman SR (2009) Overview of structural fat grafting. In: Coleman SR, Mazzola RF (eds) Fat injection from filling to regeneration. Quality Medical Publishing, St. Louis, MO, pp 93-110

3. Galiè M, Pignatti M, Scambi I, Sbarbati A, Rigotti G (2008) Comparison of different centrifugation protocols for the best yield of adipose-derived stromal cells from lipoaspirates. Plast Reconstr Surg 122(6):233e-234e

4. Peroni D, Scambi I, Pasini A, Lisi V, Bifari F, Krampera M, Rigotti G, Sbarbati A, Galiè M (2008) Stem molecular signature of adipose-derived stromal cells. Exp Cell Res 314(3):603-615

5. Rigotti G, Marchi A, Galiè M, Baroni G, Benati D, Krampera M, Pasini A, Sbarbati A (2007) Clinical treatment of radiotherapy tissue damage by lipoaspirate transplant: a healing process mediated by adipose derived adult stem cells (ASCs). Plast Reconstr Surg 119:1409-1422

6. Caplan AI, Dennis JE (2006) Mesenchymal stem cells as trophic mediators. J Cell Biochem 98(5):1076-1084

7. Scadden DT (2006) The stem-cell niche as an entity of action. Nature 441:1075-1079 